\title{
Chronique bibliographique . La dictature empêchée. La grève de la faim des femmes de mineurs. Bolivie 1977-1978
}

Dominique VIDAL

\section{(2) OpenEdition \\ Journals}

Édition électronique

URL : http://journals.openedition.org/conflits/490

DOI : $10.4000 /$ conflits. 490

ISSN : $1777-5345$

Éditeur :

CCLS - Centre d'études sur les conflits lilberté et sécurité, L'Harmattan

\section{Édition imprimée}

Date de publication : 1 septembre 2000

ISBN : 2-7475-0572-3

ISSN : $1157-996 \mathrm{X}$

\section{Référence électronique}

Dominique VIDAL, «Chronique bibliographique . La dictature empêchée. La grève de la faim des femmes de mineurs. Bolivie 1977-1978 », Cultures \& Conflits [En ligne], 40 | hiver 2000, mis en ligne le 28 septembre 2006, consulté le 30 mars 2021. URL : http://journals.openedition.org/conflits/490 ; DOI : https://doi.org/10.4000/conflits.490

Ce document a été généré automatiquement le 30 mars 2021.

Creative Commons License 


\title{
Chronique bibliographique . La dictature empêchée. La grève de la faim des femmes de mineurs. Bolivie 1977-1978
}

\author{
Dominique VIDAL
}

1 Chronique bibliographique La dictature empêchée. La grève de la faim des femmes de mineurs. Bolivie 1977-1978 ${ }^{1}$ Dominique VIDAL

2 Le 28 décembre 1977, quatre femmes de mineurs boliviens emprisonnés commencent une grève de la faim illimitée pour obtenir du gouvernement militaire une mesure d'amnistie générale et la restauration des libertés syndicales. Moins d'un mois plus tard, non sans avoir jusqu'au dernier moment cherché à les faire céder, la dictature du général Hugo Bánzer finit par accepter leurs revendications. À travers l'analyse de ces semaines qui voient croître la mobilisation derrière les grévistes, Jean-Pierre Lavaud fournit une contribution originale aux débats les plus récents en sociologie de l'action collective et de la protestation. Bien qu'entrant de plain-pied dans le champ des études sur la défense des droits de l'homme et les luttes contre l'autoritarisme en Amérique latine, son livre se veut avant tout une "sociologie de l'événement " qui, en analysant cette grève pour elle-même, entend «rendre modeste et ramener à sa juste place le savoir sociologique » (p. 12). Cette modestie est d'abord celle de l'auteur qui reconnaît ne pouvoir faire l'histoire de la grève de la faim, faute d'une documentation suffisante, mais seulement émettre quelques hypothèses sur son succès à partir du dépouillement de la presse de l'époque, des ouvrages ou des articles qui en ont parlé et d'entretiens réalisés en 1995 et 1996, bien après les faits. Les problèmes théoriques et méthodologiques posés par l'analyse sociologique de ce type de protestation sont au demeurant tels que celui qui s'y risque avec rigueur a tôt fait de voir qu'il n'y a pas ici place pour des lois générales, capables d'expliquer la naissance et le déroulement du processus de mobilisation. L'incertain, l'indéterminé, l'hésitation y sont, au contraire, constamment à l'œuvre et le sociologue n'a guère d'autre choix que de considérer 
chaque moment de l'événement étudié dans sa singularité. Cela suppose, en premier lieu, de ne jamais dissocier l'étude du niveau macrosociologique - le contexte sociopolitique dans lequel s'inscrit la protestation collective - de celle du niveau microsociologique - le système d'interaction de ses protagonistes. Cela oblige également à s'interroger en permanence sur les intentions et les décisions des acteurs. Aussi, tout au long du raisonnement, le lecteur est-il tenu informé des difficultés rencontrées et des solutions qui y ont été apportées. Il s'agit sans doute là de l'une des principales qualités de l'ouvrage où l'on suit, pas à pas, la progression de l'analyse. A cette fin, Jean-Pierre Lavaud choisit d'ailleurs, plutôt que de les réfuter comme incapables de rendre compte de la protestation considérée, de discuter patiemment les grandes théories de l'action collective et des travaux moins connus sur la grève de la faim. Il en montre à chaque fois les apports comme les limites dans l'explication des différents moments de la mobilisation. Ce dialogue permanent avec les auteurs qui nous fait croiser les thèmes majeurs de ce champ de recherches est un autre aspect de la modestie du sociologue à laquelle le lecteur est convié. L'architecture de l'ouvrage et la forme d'écriture choisie procèdent de ce même souci d'administration de la preuve : «(...) il faut comprendre que le livre est bâti comme une démonstration. Il s'agit d'articuler un ensemble de propositions qui aboutissent à un quasi-modèle explicatif de la réussite de la grève de la faim ; et donc, préalablement ou chemin faisant, de montrer la validité de ces propositions » (p. 23). Un projet qui explique l'importance accordée à la narration des contextes d'action, des événements et des récits des acteurs, et qui fait que ce qui de prime abord pourrait n'apparaître que comme d'inutiles longueurs, se révèle en réalité parfaitement justifié par les nécessités du mode d'argumentation proposé. C'est donc fort logiquement que Jean-Pierre Lavaud consacre son premier chapitre à une présentation de l'environnement social et politique dans lequel naît la protestation, une présentation didactique qui fait clairement apparaitre les principales variables à partir desquels raisonnent les sociologues de l'action collective. La Bolivie de la fin des années soixante-dix est tout d'abord une société qui connaît un type particulier de segmentation. Au relatif isolement des différents îlots de population, aux nombreux particularismes locaux et à la division du monde rural en deux grands ensembles linguistiques, s'ajoute une coupure nette entre les élites et le reste de la population. Et cette segmentation apparait d'autant plus insupportable à ceux qui la subissent que la mobilisation sociale (au sens de Karl Deutsch) commencée avec la révolution de 1952 n'a toujours pas pris fin. De plus, la grève de la faim intervient à un moment où le pays connaît une "structure des occasions politiques", selon l'expression de Sidney Tarrow, particulièrement favorable aux mouvements protestataires en raison, notamment, des divisions qui traversent l'armée et de la pression démocratisante exercée par la diffusion du discours des droits de l'Homme, soutenue par l'épiscopat latino-américain et le gouvernement de Jimmy Carter. Le second chapitre s'intéresse à un problème classique dans l'étude des mobilisations, celui du démarrage de l'action protestataire, et analyse longuement les motivations et la situation personnelle des grévistes de la faim, la nature des soutiens dont elles disposent et la spécificité du contexte dans lequel commence le jeûne. Les ressources que les deux camps en présence mobilisent pour enrayer ou renforcer le processus de mobilisation font l'objet d'un troisième chapitre. Y sont minutieusement examinés les " parades gouvernementales » (offrir des bénéfices privés et diviser, isoler, discréditer, menacer, provoquer, manifester) et les « défis des grévistes » (accéder aux médias, faire l'information, gagner la bataille morale) qui ponctuent le conflit. Discours, manœuvres, 
inflexions, coups tactiques, tout semble bon "pour imposer à l'adversaire à la fois le tempo, l'espace et le mode de résolution du conflit, en même temps que la vision de l'analyse de la situation qui mènent au conflit, et celles du déroulement de celui-ci » (p. 122). Il en ressort que jamais rien n'est joué d'avance, et que, jusqu'au dernier moment, l'issue peut basculer d'un côté comme de l'autre. Un dernier chapitre, enfin, étudie précisément les négociations qui conduisent au dénouement du conflit par un accord entre les grévistes et le gouvernement. Ce travail de reconstitution révèle, là encore, les hésitations qui animent chaque camp et l'importance prise par ce personnage emblématique des sorties de conflit qu'est le médiateur. L'intérêt de l'ouvrage pour la sociologie de l'action collective tient aussi très largement à ce qu'il offre une analyse de ce type particulier de protestation qu'est la grève de la faim dans un autre contexte que celui de l'Amérique du Nord ou de l'Europe occidentale. Contre certaines idées reçues, Jean-Pierre Lavaud rappelle que l'arme du jeûne n'est pas une pratique récente et ne correspond pas particulièrement à la forme de l'Etat-nation moderne. Observée depuis l'Antiquité en des époques et des lieux fort éloignés, elle a connu, depuis, de nombreux usages. En Bolivie, elle occupe une place importante dans ce que Charles Tilly appelle le « répertoire d'actions collectives » d'une population. En décembre 1956, le président de la République Hernán Ziles Zuazo observe ainsi un jeûne de plusieurs journées pour imposer un train de mesures économiques impopulaires, un geste destiné à montrer qu'il acceptait lui-même de se "serrer la ceinture " qui lui valut un fort appui populaire. Mais la grève de la faim fait aussi partie dans ce pays du répertoire protestataire des femmes depuis les années cinquante. Elle y est une forme d'action qui leur permet d'agir quand d'autres moyens, notamment violents, utilisés par les hommes ont échoué. Facteur essentiel du succès du mouvement étudié, le vaste soutien rencontré dans l'opinion par les jeûneurs a en effet dépendu de leur capacité à accréditer l'idée d'une grève spontanée, non politique, soulèvement humanitaire mû par la détresse. La faiblesse des bénéfices individuels privés que ces femmes pouvaient espérer tirer de leur action et l'importance des risques pour la vie encourus rend du reste manifeste que "le simple calcul utilitaire ne peut expliquer l'engagement protestataire des initiatrices de la grève» (p. 172). L'analyse sociologique montre cependant que la décision de ces femmes d'entamer une grève de la faim n'a rien d'une décision "spontanée ", "irréfléchie ", comme le soutenaient leurs partisans contre les accusations de calcul politique du gouvernement. D'abord parce que la socialisation familiale et politique des grévistes les prédisposent à entreprendre ce type d'action. Ensuite parce que le jeûne débute dans un contexte politique où les organisations d'opposition ont peu à peu créé une situation favorable à leur engagement et à la mobilisation qui la suit. L'idéologie des droits de l'Homme fournit, en effet, un langage commun fédérateur à des acteurs de nature et d'orientations sensiblement différentes qui ont pris l'habitude de s'épauler dans les années précédant la grève. Mais portées par des femmes accompagnées de leurs enfants et soutenues par des religieux, les revendications des grévistes dépassent l'audience habituelle de ces organisations. JeanPierre Lavaud voit dans ce changement, une modification des "cadres de références " d'une partie de l'opinion publique, un "dégel cognitif» produit par la victoire des grévistes dans ce qui fut pour beaucoup une «bataille du sens » entre les tenants de la «spontanéité » de l'action protestataire et ceux qui la prétendaient «organisée » de longue date par les organisations politiques d'opposition. Si l'on sait gré à l'auteur d'avoir su proposer une analyse sociologique de l'action de ces Boliviennes sans tomber dans les égarements idéologiques dans lesquels sombre souvent la littérature sur 
l'engagement politique des femmes, on peut toutefois regretter qu'il n'évoque pour ainsi dire pas la question de la place des femmes dans le monde andin. On aurait par exemple aimé savoir si leur protestation pouvait être interprétée comme la prolongation d'un rôle traditionnel de défense du foyer quand les hommes sont privés des moyens de subvenir à ces besoins ou représentait un changement notable dans les rapports sociaux de sexe. Ce regret n'enlève néanmoins rien à la portée de l'ouvrage et à son caractère particulièrement stimulant. D'autant que son auteur suggère implicitement un programme de recherches sur ce type d'action protestataire en soulignant maintes fois les questions qu'une documentation lacunaire contraint à laisser sans réponses ou l'apport qu'aurait représenté une enquête de terrain à chaud.

\section{NOTES}

1. Jean-Pierre Lavaud, La dictature empêchée. La grève de la faim des femmes de mineurs. Bolivie 1977-1978, Paris, Editions du CNRS, 1999.

\section{INDEX}

Index géographique : Amérique du Sud, Bolivie

Mots-clés : dictature, genre, résistances politiques

Index chronologique : 1970 - 1980 